Target journal: Supportive Care in Cancer.

\title{
Prevalence of ethical dilemmas in advanced cancer patients (secondary analysis of the PALCOM study)
}

Tuca A, Viladot M, Barrera C, Chicote M, Casablancas I, Cruz C, Font E, MarcoHernández J, Padrosa J, Pascual A, Codorniu N, Román B.

Albert Tuca, ORCID iD 0000-0002-4633-5856, Hospital Clínic de Barcelona, Barcelona, Spain.ATUCA@clinic.cat

Margarita Viladot, Hospital Clínic de Barcelona, Barcelona, Spain. VILADOT@clinic.cat

Carmen Barrera, Hospital Clínic de Barcelona, Barcelona, Spain. MBARRERA@clinic.cat

Manoli Chicote, Hospital Clínic de Barcelona, Barcelona, Spain. MCHICOTE@clinic.cat

Irene Casablancas, Hospital Clínic de Barcelona, Barcelona, Spain. ICASABLANCA@clinic.cat

Claudia Cruz, Hospital Clínic de Barcelona, Barcelona, Spain.CACRUZ@clinic.cat

Elena Font, Hospital Clínic de Barcelona, Barcelona, Spain. EFONT@clinic.cat

Javier Marco-Hernández, Hospital Clínic de Barcelona, Barcelona, Spain. JMARCO@clinic.cat.

Joan Padrosa, Hospital Clínic de Barcelona, Barcelona, Spain. PADROSA@clinic.cat

Anais Pacual, Hospital Clínic de Barcelona, Barcelona, Spain. ALPASCUAL@clinic.cat

Núria Codorniu, Fundación Atención a la Dependencia Sant Joan de Deu, Barcelona, Spain.Nursing Schooll of University of Barcelona.n.codorniu@fad-sjd.org

Begoña Román iD 0000-0001-6090-0172, Faculty of Philosophy of the Univerity of Barcelona, Spain. broman@ub.edu

Author for correspondence:

Albert Tuca, ORCID iD 0000-0002-4633-5856, Hospital Clínic de Barcelona, Barcelona, Spain.ATUCA@clinic.cat

\section{Declarations}


Funding: Not applicable.

\section{Conflicts of interest/Competing interests:}

The authors declare that they have no conflicts of interest.

\section{Ethics approval:}

All procedures were performed according to the legislation for this study type. The study was conducted according to the Declaration of Helsinki, the Data Protection Directive, and local legislative requirements. The study was approved by the Clinical Investigation Ethics Committee of Hospital Clínic, Barcelona, Spain.

\section{Consent to participate:}

All patients included in the study gave their written informed consent at the baseline visit, before data collection.

\section{Consent for publication:}

Not applicable.

\section{Available of data and material:}

Additional data are available on request.

\section{Code availability:}

Not applicable.

\section{Acknowledgments:}

Writing and editorial assistance was provided by Content Ed Net (Madrid, Spain).

\section{Author contributions:}

Tuca A was the study coordinator and was involved in the study design, protocol, preparation of the case report form, and data analysis. Viladot M, Barrera C, and Chicote $\mathrm{M}$ were involved in study design, recruitment, data collection, and analysis. Casablancas I, Cruz C, Font E, Marco-Hernández J, Padrosa J, and Pascual A were involved in data analysis. Codorniu $\mathrm{N}$ and Román $\mathrm{B}$ were involved as ethics specialists in data analysis and in the qualitative discussion of the study results.

The first draft of the manuscript was written by Tuca A, and all authors reviewed and commented on provisional versions of the manuscript. All authors read and approved the final version of the manuscript.

\section{Abstract}




\section{Purpose}

The main aim of this study was to determine the prevalence of ethical dilemmas in the end-of-life process in advanced cancer patients.

Methods

We carried out a multicenter, cross-sectional, observational, prospective study in a cohort of cancer patients whose life expectancy was $\leq 6$ months. We recorded sociodemographic characteristics, diagnosis of cancer, symptom burden, cognitive and functional status, emotional impact, and sociofamilial risk factors. The main outcome measure was the detection of ethical dilemmas, based on the following definition: Conflict in decisionmaking during the end-of-life process that involves the need to choose between morally acceptable opposing options, where none is clearly preferable to another.

Results

We included 324 patients (mean age, 69 years; 58\% men). We identified 117 dilemmas in 90 patients $(27.8 \%)$. The dilemmas detected were as follows: a) conflicts of information (adaptive denial, conspiracy of silence, information exceeding patient's desired limit), $15.7 \%$; b) discrepancies in proportionality (discussion on futility, rejection of treatment, withdrawal of life support measures), $16.7 \%$; c) unrealistic expectations about the outcome of clinical trials, $2.5 \%$; d) request for euthanasia or medically assisted suicide, $1.2 \%$. We observed a greater prevalence of ethical dilemmas in men, in patients receiving active cancer treatment, and in patients with emotional distress $(p<0.05)$.

Conclusions

Prevalence of ethical dilemmas during the end-of-life process in cancer patients is relevant. Most dilemmas were associated directly or indirectly with respect for patient autonomy. In this context, the communication skills of the health professionals and advanced care planning take on a key role.

Key words: Cancer. End of life. Ethical dilemma. Conflict. Decision-making.

\section{Introduction}


Caring for patients with incurable cancer and relieving their pain and suffering is as important in ethical terms as preventing disease, maintaining good health, and avoiding premature death [1].

Incurable disease and the threat of imminent death constitute a unique life crisis. Symptom burden, emotional impact, spirituality, religious beliefs, moral values, life history, and sociocultural context are key elements for the patient and the patient's family during the process of coping with the end of life. This life experience is complex, because it depends on the interaction between these variables in an equilibrium which is not always stable or predictable [2]. End-of-life care must bring together these essential needs with the aim of maximizing quality-of-life and preserving patients' dignity [3-6].

In the complex end-of-life scenario, patients, their families, and the care team actively participate in the decision-making process in accordance with their knowledge and moral values. The widely accepted ethical principles of beneficence, nonmaleficence, justice, and autonomy govern the actions of health care professionals [7]. However, moral discrepancies often arise when decisions are shared between the various parties involved in the end-of-life process. By definition, an ethical dilemma is a conflict in the making of decisions between various options with differing moral content, none of which is unequivocally preferable in the opinion of those who analyze the dilemma [8].

Multiple publications provide an in-depth study of the ethical aspects of the end-of-life process from a theoretical standpoint [8-13]; however, we do not know the prevalence of dilemmas in daily clinical practice when they constitute a problem for decision-making. The objective of this study was to determine the prevalence of ethical dilemmas during the end-of-life process in advanced cancer patients.

\section{Methods}

We carried out a multicenter, cross-sectional, observational, prospective study. The present study is a secondary analysis of a wider project (the PALCOM study), whose objective was to identify those factors that come into play when we attempt to determine the complexity of palliative requirements in advanced cancer patients [2].

All public medical oncology, primary care, and home palliative care centers, as well as medium- to long-stay units, from the health district of Eixample Esquerra, Barcelona, Spain were invited to participate in the study.

Between November 2012 and January 2013, the participating centers consecutively included all patients who fulfilled the following inclusion criteria: age $\geq 18$ years, diagnosis of cancer, life expectancy $\leq 6$ months, and agreement to participate through informed consent.

An ad hoc multidimensional questionnaire based on the recommendations of the European Society of Palliative Care [14] was designed to determine the characteristics of the study population. The questionnaire was used to record the following: sociodemographic data; origin and extension of the cancer; general/functional status (Karnofsky Performance Status [KPS] scale and Barthel index); symptom burden (intensity of pain, asthenia, anorexia, nausea and vomiting, constipation, dyspnea, somnolence, insomnia, anxiety and depression, using an 11-point numeric rating scale 
[NRS], where zero is no pain and 10 is the worst pain imaginable); cognitive status (Confusion Assessment Method) [15]; classification of pain (Edmonton Classification System for Cancer Pain) [16]; and sociofamilial risk factors. In the case of patients with cognitive impairment, symptoms were evaluated by family members or the legal representative. Maximum follow-up was 6 months, and the date and place of death were recorded.

The main outcome measure was the presence of ethical dilemmas, evaluated during the first study visit and in accordance with a working definition that was agreed upon by the research team.

\section{Definition of ethical dilemma}

The definition of ethical dilemma in the end-of-life process, as agreed upon by the research team, was as follows: "Conflict in decision making during the end-of-life process that includes the need to choose between morally acceptable opposing options, where none is clearly preferable to another."

Similarly, with the aim of harmonizing the data recorded, we established a literaturebased list [8-13] of possible ethical dilemmas (patient information, proportionality of healthcare, clinical research, palliative sedation, and rational desire to bring death forward or an explicit request for assisted suicide or euthanasia).

Good clinical practice is governed by the basic principles of bioethics (autonomy, beneficence, nonmaleficence, and justice) [7]. The question of ethics arises when the automatic response to patient care problems, which is based on the principles of bioethics, is questionable in a specific situation in clinical practice. Dilemmas usually arise when there is a conflict between ethical principles and it is difficult to establish a hierarchy of one over another.

After the multidimensional evaluation, the investigator recorded and classified the ethical dilemmas observed. All the investigators received specific training in the examination of ethical dilemmas, which included a review of the following topics: agreed definition of ethical dilemmas, common ethical conflicts and their categorization based on the literature [8-13], and skills necessary to detect, explore, and record these dilemmas in this study. Once the quantitative results were known, the research team participated in a moderated discussion group to identify and standardize the key ideas related to the conflicts observed in each category.

The research team participated in the analysis of the quantitative results and in the narrative description of the dilemmas observed.

\section{Statistical Analysis}

Data were analyzed with the aim of describing the characteristics of the study sample and determining the prevalence of ethical dilemmas, both in absolute terms and in terms of predefined categories. Categorical or dichotomous variables were analyzed using absolute and relative frequencies. Continuous variables were expressed using a measure of central tendency (mean or median) and dispersion (standard deviation), with a $95 \%$ confidence interval $(95 \% \mathrm{CI})$. We also compared the prevalence of ethical dilemmas by sex, active cancer treatment, general deterioration (KPS scale $\leq 50 \%$ ), and intense 
emotional distress (NRS anxiety + depression $\geq 10$ ). Categorical variables were compared using the chi-square test or Fisher exact test; continuous variables were compared using the $t$ test or Wilcoxon test. Statistical significance was set at $p<0.05$. The statistical analysis was carried out using $\mathrm{R}$ version 3.1.2.

The estimated sample size was at least 300 patients, according to data from previous studies by our group $[2,17]$ and taking into account the reasonable maximum capacity of the participating centers and the fact that variables were to be recorded under conditions of daily clinical practice.

\section{Ethics}

Before being included in the study, patients received an information leaflet and gave their written informed consent to participate. In the case of patients with cognitive impairment that prevented them from understanding the study, we sought the authorization of a family member or legal representative. The study was approved by the Clinical Investigation Ethics Committee of Hospital Clínic, Barcelona, Spain.

\section{Results}

Twenty-four centers participated in the study (16 primary care centers, 3 hospitals, 3 home palliative care teams, and 2 medium- to long-stay units). A total of 324 patients met the inclusion criteria during the study period. All patients agreed to participate and signed the informed consent document (181 in hospitals [55.8\%], 71 in home palliative care units [21.9\%], 44 in medium- to long-term-stay units [13.6\%], and 28 in primary care centers [8.6\%]) (Figure 1). Mean age was $69( \pm 12)$ years, and 58\% were men. The most common primary origin was lung cancer [22.2\%]). A total of 272 patients $(83.9 \%)$ were receiving active cancer treatment. Most patients had moderate functional impairment (mean Barthel index, $60.5[ \pm 26])$, and $129(39.8 \%)$ were highly dependent (KPS $\leq 50 \%)$. The main number of symptoms per patient was $5.9( \pm 1.2)$, and $26.8 \%$ had a considerable symptom burden ( $\geq 5$ symptoms with NRS $\geq 4$ ). Cognitive impairment was observed in 25 patients (7.7\%). At least 1 sociofamilial risk factor was reported in 222 cases $(68.5 \%)$. Patient characteristics according to the multidimensional evaluation are summarized in Table 1.

The multidimensional evaluation revealed at least 1 ethical dilemma in 90 patients (27.8\%). A total of 117 ethical dilemmas were recorded (mean, 1.3/patient $[ \pm 0.7]$ ). The prevalence of dilemmas according to the pre-established categories was as follows: conflicts associated with information provided to the patient and/or the patient's family, $51(15.7 \%)$; discrepancies in therapeutic proportionality within the patient's family, 26 (8\%); disagreement on decisions on therapeutic proportionality between the care team and the patient and/or the patient's family, 14 (4.3\%); discrepancies with regard to therapeutic proportionality within the team or between health care professionals, 12 (3.7\%); disagreement associated with clinical trials, 8 (2.5\%); request for euthanasia or assisted suicide, 4 (1.2\%); and disagreement with respect to the withdrawal of life support measures, $2(0.6 \%)$. In this study, we did not record any ethical dilemmas specifically associated with the indication for palliative sedation.

The mean age of patients with ethical dilemmas was $67.9( \pm 7)$ years, and there were no significant differences compared with the overall age of the sample. The prevalence of dilemmas was significantly greater in men $(66.7 \%)$, in patients receiving active cancer 
treatment $(61.1 \%)$, and in patients with intense emotional distress $(73.3 \%)(p<0.05)$ (Table 2). We also observed a trend toward a greater prevalence of discrepancies between the care team and the patient/family with respect to intense functional impairment $(85.7 \%)$, although this was not significant, probably owing to the sample size $(p=0.075)$. At 6 months of follow-up, 202 patients were evaluable (62.3\%); of these, $177(87.6 \%)$ had died. There were no significant differences for the probability of death in hospital between the overall sample $(33.2 \%)$ and the patients who presented ethical dilemmas $(35 \%)$.

\section{Discussion}

The objective of this study is particularly relevant because, although several articles have addressed the theoretical aspects of ethical dilemmas in patients with advanced cancer, there are no data on prevalence in daily clinical practice [18].

We believe that the study population is representative, since the sociodemographic data, prevalence of primary cancer, and symptom burden are comparable with data from previous studies and population-based cancer registries [2,17-19].

The first difficulty when designing this study was that of deciding on the definition of an ethical dilemma. It is obvious that such a definition could affect prevalence data. The agreed definition of ethical dilemma in this study focused on situations characterized by a moral conflict between divergent positions of uncertain resolution where it was imperative to choose one. After the analysis of the results, the research team confirmed the appropriateness of the definition and did not detect conflicts that could not be included in the definition or in the categories established.

This study confirms that the prevalence of ethical dilemmas is relevant $(27.8 \%)$. The likelihood of an ethical dilemma arising was significantly greater in men, patients receiving active treatment for cancer, and patients with intense emotional distress.

Other than providing speculative reasons, we were unable to explain the greater prevalence of ethical dilemmas in men. Maintaining or suspending active treatment during the last months of life in cancer patients must be evaluated very carefully, taking into account both the expected increase in survival and the expected quality of life. In such a situation, shared decision-making can lead to conflicts, which are sometimes difficult to resolve and are associated with therapeutic proportionality or the adaptation of information to realistic expectations.

We believe that the greatest prevalence of ethical dilemmas in patients with intense emotional distress is due to the fact that a clinical situation of uncertain resolution can itself have a considerable emotional impact and that a poorly adapted coping strategy seriously hampers decision-making.

Respecting a person's dignity requires respect for his/her autonomy, and there can be no autonomy without appropriate information $[4,5]$. Patients have the right to adequate and truthful information so that they can decide freely on their health and on available clinical options [21-23]. However, some authors and ethics codes understand that the inalienable right to information does not necessarily require patients to be fully informed in any circumstances and against their wishes [24]. Consequently, the means of providing this 
information, in terms of both specificity and quantity, should be tailored to the needs and preferences of the patient $[22,25]$. In this asymmetrical relationship between the patient and the care team, training in communication skills for health professionals is essential.

In this study, $16 \%$ of patients had a dilemma associated with the information provided, which, through excess or lack, hampered appropriate decision-making. The investigators identified 3 types of conflict: a) information exceeding patient's desired limit; b) adaptive denial; c) conspiracy of silence. Information exceeding patient's desired limit was described as information provided quickly in all its aspects without considering the opinion of the patient in his/her specific circumstances and that hampered calm decisionmaking because of a high emotional impact. On the other hand, respecting the limit in the information explicitly stated by the patient can constitute an ethical dilemma, since this may distort decision-making shared with health professionals. Denial was described as an adaptive process, in which the patient preferred not to know all the aspects of the disease, especially the prognosis, or to adopt alternative reasoning and which, at the time of the evaluation, limited appropriate decision-making. Conspiracy of silence was defined as the agreement of the patient's family to hide or partially or totally modify information on the disease, especially the prognosis, with compassionate intent and based on the conviction that the patient did not have sufficient emotional resources to cope with a life threat $[25,26]$. In this context, the family asks the care team to respect and to collaborate in the conspiracy of silence. Management of a conspiracy of silence is a difficult challenge for care teams and should clearly include both the patient and the patient's family. The objective with respect to the patient is to provide additional information prudently and respectfully until a sufficient amount of information has been provided to enable autonomous decision-making, including the limits of the information the patient wishes to receive. In the case of the family, the objectives would be to change a paternalistic and compassionate attitude of silence for calm and reassuring company for the patient. Throughout this process, the necessary psychological support should be provided for both the patient and the family. Finally, an agreement should be made to respect the limits that the patient sets on the quantity and intensity of the information. In our opinion, adaptive denial and the conspiracy of silence more often cause ethical dilemmas than information that exceeds the limit set by the patient.

Various studies report that between $20 \%$ and $38 \%$ of patients with terminal advanced cancer have not talked about the prognosis of the disease at any time [27-31]. While no specific reason is given in the studies, it is possible that in most cases the reason is adaptive denial or conspiracy of silence. The difference between these prevalence values and that observed in the present study ( $20 \%$ to $38 \%$ versus $16 \%$ ) could be due to the fact that in these publications, the level of information was recorded, whereas in our study we identified conflicts in shared decision-making associated with the amount of information the patient had received.

We observed ethical dilemmas related to proportionality in $17 \%$ of patients, for whom the investigators identified 2 different conflicts: a) discrepancies concerning the determination of futility of the diagnostic or therapeutic options; b) rejection of treatment.

Futility refers to a medical act that is considered useless because the desired result, based on the best evidence available, is very unlikely, and provides no reasonable benefit for 
the patient [31-35]. It is a complex concept, because it focuses on the time remaining, as well as on the quality of the life experience and the potential benefit or harm to the patient of interventions aimed at a hypothetical but improbable prolongation of survival. Both objective knowledge of the disease and treatment options and the value system and personal experiences of all the participants (care team, patient/family) are essential elements of the discussion of futility in a specific clinical situation [35].

The rejection of a treatment that the care team considers appropriate, based on a rational and reflective choice, is not ethically questionable if the conditions of autonomy are maintained (competence, information, absence of external pressure, responsibility for the extent of the decisions) [21-23]. However, some patients explained that after the insistence of their physicians, they accepted treatment options about which they were unsure because of fear of medical abandonment. On the other hand, some professionals believe that insisting on an effective and safe treatment is an ethical imperative and that accepting a patient's wish not to receive therapy without trying to persuade him or her could be considered a maleficent act. Therefore, the skill of the healthcare professional lies in the balance between persuasion with respect to the measures that he/she considers appropriate and rigorous respect for the patient's autonomous decisions so that he/she never feels medically abandoned.

Ethical dilemmas associated with medical research, mainly in clinical trials, were detected in $2.5 \%$ of the patients included. All of the patients with cancer included in clinical trials agreed to participate freely after receiving appropriate information. However, the investigators identified as an ethical conflict those cases in which the patient's expectations did not fully correspond with the real expected outcomes of the clinical trial.

The rational choice of bringing death forward by means of euthanasia or medically assisted suicide was an insurmountable legal problem in countries where these practices are not authorized, irrespective of the moral value that stakeholders attribute to this issue. It is noteworthy that the request to bring death forward in the present study (1.2\%), which was carried out in Spain, is similar to that observed in countries where euthanasia is legal $(0.7-2.8 \%)[36,37]$.

Terminal sedation refers to the deliberate administration of sedatives at doses and in combinations necessary to reduce the level of consciousness of a terminally ill patient for as long as necessary for appropriate relief of 1 or more refractory symptoms [38-40]. Approximately $25 \%(10 \%-50 \%)$ of advanced cancer patients require palliative sedation at the end of their lives [40]. Currently, there are no doubts about the theoretical legitimacy of palliative sedation and the ethical criteria that differentiate it from euthanasia [39]. However, in daily clinical practice, there may arise dilemmas associated with interpretation of the ethical requirements underlying palliative sedation: presence of refractory symptoms; drug doses that are proportional to the objective of lowering the patient's level of consciousness and not to that of reducing the time remaining; patient participation (explicit, implicit, or delegated consent) [8-10]. In this study, we did not find any cases of ethical dilemmas associated with terminal sedation, probably because in most cases, life expectancy was measured in months. Similarly, we observed no cases where palliative sedation was called into question as a concept. Possible discrepancies 
concerning the indication of palliative sedation in a specific situation or advanced care plan in this study were included in the category of dilemmas of proportionality.

Most of the dilemmas observed in this study were associated, either directly or indirectly, with respect for patient autonomy. Advance care planning is a formal process of reflective discussion between the patient, his/her family, and the healthcare team about values, goals, and wishes related to current and future healthcare. The available literature confirms that advanced care planning improves communication between the patient, his/her family, and the healthcare team, facilitates shared decisions, and documents wishes with respect to the intensity of care in the end-of-life process and the place of death [41]. We believe that advanced care planning could probably have reduced the incidence and magnitude of many of the ethical dilemmas observed in this study.

The main limitation of the study is that the identification and evaluation of the dilemmas often depended on the subjective criteria of the investigators. Furthermore, this is a crosssectional study that defines prevalence within a pre-established timeframe. It does not explore the dilemmas that might arise during the natural history of a patient's disease. The ethical argument could be considerably influenced by socioeconomic and cultural aspects. In addition, since this study was performed in an urban setting in a developed country with a universal public health system, we do not know if the data can be extrapolated to other population types. In this study, loss to follow-up at 6 months was significant (37.6\%), mainly due to difficulties in contact with patients at the end of life or with grieving relatives. However, loss to follow-up did not affect the assessment of the main study variable (identification and classification of the ethical dilemma), since this was recorded at the baseline visit. It is also noteworthy that this study only recorded the prevalence of consistent ethical conflicts that significantly hindered the decision-making process; it did not explore the prevalence of the need to use ethical arguments in daily clinical practice, which is obviously more pronounced.

Based on the data of this study, we consider that it would be important to develop future lines of research on the incidence of ethical dilemmas resulting from the diagnosis at any stage of cancer, as well as research into the impact of advanced care planning on the frequency and magnitude of these dilemmas.

\section{Conclusions}

Our data enable us to conclude that the prevalence of ethical dilemmas during the end-oflife process in patients with cancer is relevant. Most of the dilemmas observed were associated, either directly or indirectly, with respect for patient autonomy. In this context, the communication skills of the health professionals and advanced care planning take on a key role. 


\section{Figure 1: Study flow diagram}




\section{References}

1. Hastings Center Report (1996) The goals of Medicine. Setting New Priorities. 26 (6):S1-S27.

2. Tuca A, Gómez-Martínez M, Prat A (2018) Predictive model of complexity in early palliative care: a cohort of advanced cancer patients (PALCOM study). Support Care Cancer 26(1):241-249. doi: 10.1007/s00520-017-3840-3

3. Maté-Méndez J, González-Barboteo J, Calsina-Berna A et al (2013) The Institut Català d'Oncologia model of palliative care: an integrated and comprehensive framework to address the essential needs of patients with advanced cancer. J Palliat Care 29(4):237-243

4. Chochinov HM (2007) Dignity and the essence of medicine: The A, B, C, and D of dignity conserving care. BMJ 335(7612):184-187. doi: 10.1136/bmj.39244.650926.47

5. Chochinov HM (2013) Dignity in care: time to take action. J Pain Symptom Manage;46(5):756-759. doi: 10.1016/j.jpainsymman.2013.08.004

6. Hui D, Hannon BL, Zimmermann C, Bruera E (2018) Improving patient and caregiver outcomes in oncology: Team-based, timely, and targeted palliative care. CA Cancer J Clin 68(5):356-376. doi: 10.3322/caac.21490

7. Beauchamp T, Childress J (2001). Moral Norms. In: Principles of Biomedical Ethics, 5th ed. Oxford University Press, New York, pp 1-25.

8. Ong WY, Yee CM, Lee A (2012) Ethical dilemmas in the care of cancer patients near the end of life. Singapore Med J 53(1): 11-16

9. Chih AH, Su P, Hu WY, Yao CA et al (2016) The Changes of Ethical Dilemmas in Palliative Care. A Lesson Learned from Comparison Between 1998 and 2013 in Taiwan. Medicine (Baltimore) 95(1):e2323. doi: 10.1097/MD.0000000000002323

10. Huang HL, Yao CA, Hu WY et al (2018) Prevailing Ethical Dilemmas Encountered by Physicians in Terminal Cancer Care Changed After the Enactment of the Natural Death Act: 15 Years' Follow-up Survey. J Pain Symptom Manage 55(3):843-850. doi: 10.1016/j.jpainsymman.2017.11.033

11. Guevara-López U, Altamirano-Bustamante MM, Viesca-Treviño C (2015) New frontiers in the future of palliative care: real-world bioethical dilemmas and axiology of clinical practice. BMC Med Ethics 16:11. doi:10.1186/s12910-0150003-2

12. Chiu TY, Hu WY, Huang HL, Yao CA, Chen CY (2009) Prevailing ethical dilemmas in terminal care for patients with cancer in Taiwan. J Clin Oncol 27(24):3964-3968. doi: 10.1200/JCO.2008.21.4643

13. Kinzbrunner BM (1995) Ethical dilemmas in hospice and palliative care. Support Care Cancer 3(1):28-36. doi: 10.1007/BF00343918

14. Sigurdardottir KR, Kaasa S, Rosland JH, Bausewein C, Radbruch L, Haugen DF, PRISMA (2014) The European Association for Palliative Care basic dataset to describe a palliative care cancer population: Results from an international Delphi process. Palliat Med 28(6):463-473. doi: 10.1177/0269216314521264 
15. van Velthuijsen EL, Zwakhalen SMG, Warnier RM, Mulder WJ, Verhey FRJ, Kempen GIJM (2016) Psychometric properties and feasibility of instruments for the detection of delirium in older hospitalized patients: a systematic review. Int $\mathrm{J}$ Geriatr Psychiatry 31(9):974-989. doi: 10.1002/gps.4441

16. Fainsinger RL, Nekolaichuk CL (2008) A "TNM" classification system for cancer pain: the Edmonton Classification System for Cancer Pain (ECS-CP). Support Care Cancer 16(6):547-555. doi: 10.1007/s00520-008-0423-3

17. Tuca-Rodriguez A, Gómez-Batiste X, Espinosa-Rojas J, Martínez-Muñoz M, Codorniu N, Porta-Sales J (2012) Structure, organization and clinical outcomes in cancer patients of hospital support teams in Spain. BMJ Support Palliat Care 2(4):356-362. doi: 10.1136/bmjspcare-2011-000083

18. Schofield G, Brangan E, Dittborn M et al (2019) Real-world ethics in palliative care: protocol for a systematic review of the ethical challenges reported by specialist palliative care practitioners in their clinical practice. BMJ Open 9(5):e028480. doi: 10.1136/bmjopen-2018-028480

19. Oechsle K1, Goerth K, Bokemeyer C, Mehnert A (2013) Symptom burden in palliative care patients: perspectives of patients, their family caregivers, and their attending physicians. Support Care Cancer 21(7):1955-1962. doi: $10.1007 / \mathrm{s} 00520-013-1747-1$

20. Globocan. International Agency for Research in Cancer. World Health Organization. Cancer Today. Estimated number of deaths, both sexes, worldwide in 2012. Available at: http:/gco.iarc.fr/today/online-analysis-triangle? mode $=$ cancer\&mode_population $=$ continents\&population (last accessed September 2019)

21. AMA Code of Medical Ethics Opinion 2.1.3. Available at: https://www.amaassn.org/delivering-care/ethics/withholding-information-patients (last accessed August 2019)

22. Code of Medical Ethics (2016). Organización Médica Colegial de España. Available at: https://www.cgcom.es/sites/default/files/code_of__medical_ ethics/index.html\# (Last accessed August 2019)

23. Foster C (2010) Autonomy should chair, not rule. Lancet 375(9712):368-369. doi: 10.1016/s0140-6736(10)60156-0

24. Convention for the Protection of Human Rights and Dignity of the Human Being with regard to the Application of Biology and Medicine: Convention on Human Rights and Biomedicine. Council of Europe (1997). https://www.coe.int/en/web/conventions/full-list/-/conventions/

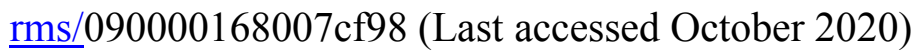

25. Lemus-Riscanevo P, Carreño-Moreno S, Arias-Rojas M (2019) Conspiracy of Silence in Palliative Care: A Concept Analysis. Indian J Palliat Care 25(1):24-29. doi: 10.4103/IJPC.IJPC_183_18

26. Ibañez-Masero O, Carmona-Rega IM, Ruiz-Fernández MD et al (2019) Communicating Health Information at the End of Life: The Caregivers' Perspectives. Int J Environ Res Public Health 16(14):2469. doi: 10.3390/ ijerph16142469 
27. Epstein AS, Prigerson HG, O'Reilly EM, Maciejewski PK (2016) Discussions of Life Expectancy and Changes in Illness Understanding in Patients with Advanced Cancer. J Clin Oncol 34(20):2398-2403. doi: 10.1200/JCO.2015.63.6696

28. Enzinger AC, Zhang B, Schrag D, Prigerson HG (2015) Outcomes of Prognostic Disclosure: Associations with Prognostic Understanding, Distress, and Relationship with Physician among Patients with Advanced Cancer. J Clin Oncol 33(32):3809-3816. doi: 10.1200/JCO.2015.61.9239

29. Justo Roll I, Simms V, Harding R (2009) Multidimensional problems among advanced cancer patients in Cuba: awareness of diagnosis is associated with better patient status. J Pain Symptom Manage 37:325-330. doi: 10.1016/j.painsymman.2008.02.015

30. Mystakidou K, Tsilika E, Parpa E, Katsouda E, Vlahos L (2005) Patterns and barriers in information disclosure between health care professionals and relatives with cancer patients in Greek society. Eur J Cancer Care (Engl) 14(2):175-181. doi: $10.1111 / \mathrm{j} .1365-2354.2005 .00554 . x$

31. Bernat JL (2005) Medical Futility. Definition, Determination, and Disputes in Critical Care. Neurocrit Care 2:198-205. doi: 10.1385/NCC:2:2:198

32. Schneiderman LJ, Jecker NS, Jonsen AR (1990) Medical futility: its meaning and ethical implications. Ann Intern Med 112:949-954. doi: 10.7326/0003-4819-11212-949

33. Schneiderman LJ, Jecker NS, Jonsen AR (1996) Medical futility: responses to critiques. Ann Intern Med 125(8):669-674. doi: 10.7326/0003-4819-125-8199610150-00007

34. Schneiderman LJ, Jecker NS (1995) Wrong Medicine: Doctors, Patients, and Futile Treatments. Johns Hopkins University Press, Baltimore

35. Kass JS, Lewis A, Rubin MA (2018) Ethical Considerations in End-of-life Care in the Face of Clinical the Face of Futility. Continuum (Minneap Minn) 24(6):1789-1793. doi: 10.1212/CON.0000000000000680

36. Cohen J, Dierickx S, Penders YWH, Deliens L, Chambaere K (2018) How accurately is euthanasia reported on death certificates in a country with legal euthanasia: a population-based study. Eur J Epidemiol 33(7):689-693. doi: 10.1007/s10654-018-0397-5

37. Onwuteaka-Philipsen BD, Brinkman-Stoppelenburg A, Penning C, de Jong-Krul GJ, van Delden JJM, van der Heide A (2012) Trends in end-of-life practices before and after the enactment of the euthanasia law in the Netherlands from 1990 to 2010: a repeated cross-sectional survey. Lancet 380(9845):908-915. doi: 10.1016/S0140-6736(12)61034-4

38. Twycross R (2019) Reflections on palliative sedation. Palliat Care 12:1178224218823511. doi: 10.1177/1178224218823511

39. Materstvedt LJ (2020) Distinction between euthanasia and palliative sedation is clear-cut. J Med Ethics 46(1):55-56. doi: 10.1136/medethics-2019-105457

40. Cherny NI; ESMO Guidelines Working Group (2014) ESMO Clinical Practice Guidelines for the management of refractory symptoms at the end of life and the use of palliative sedation. Ann Oncol Sep 25 Suppl 3:iii143-152. doi: 10.1093/annonc/mdu238. 
41. Jimenez G, Tan WS, Virk AK, Low CK, Car J, Ho AHY (2018) Overview of Systematic Reviews of Advance Care Planning: Summary of Evidence and Global Lessons. J Pain Symptom Manage. 56(3):436-459.e25. doi:10.1016/j.jpainsymman.2018.05. 016. 


\section{Figure 1: Study flow diagram}

Eligible advanced cancer patients with expected survival $\leq 6$ months ( $N=324)$

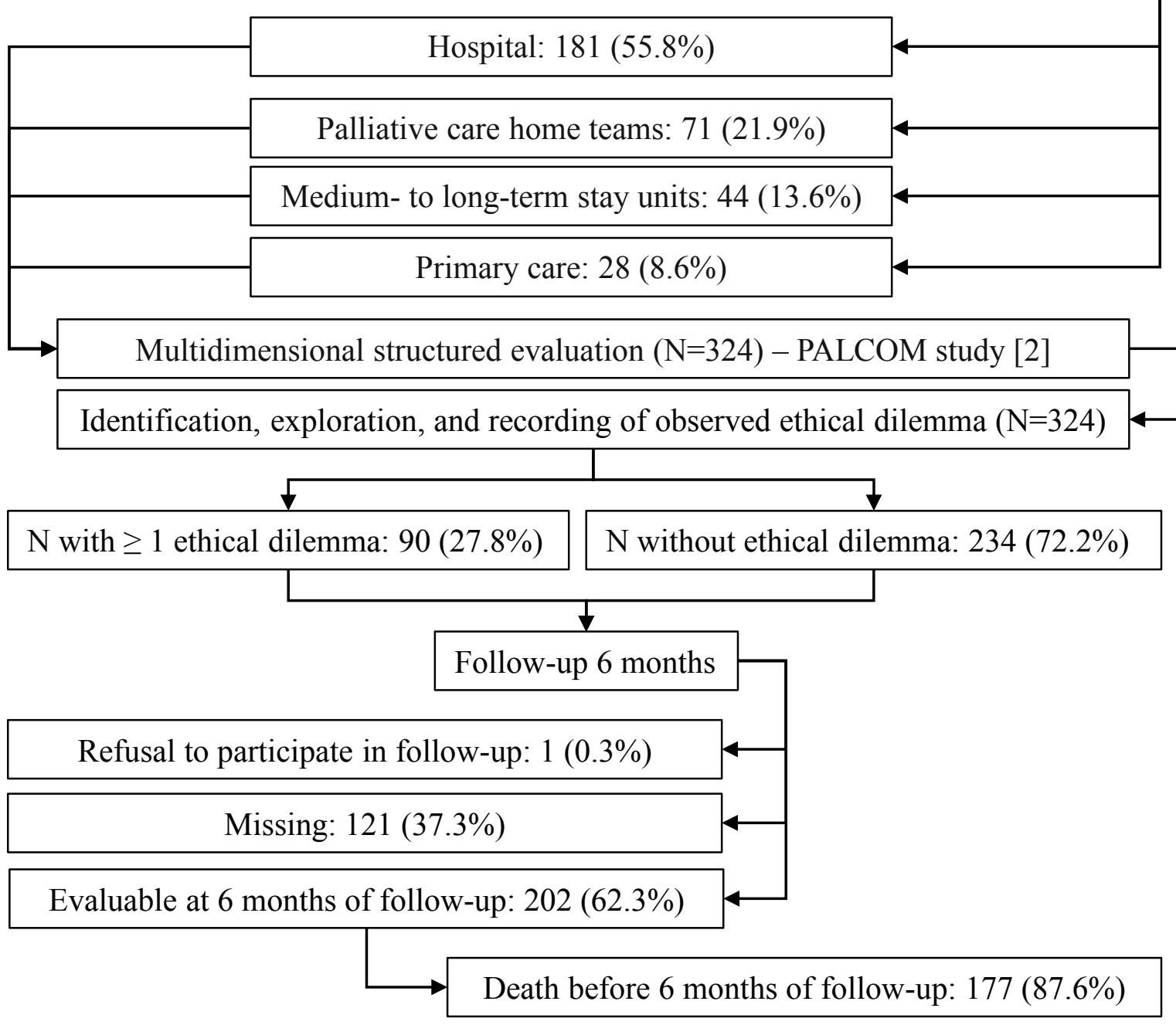




\begin{tabular}{|c|c|c|}
\hline Total $n$ & & 324 \\
\hline Sex & $\operatorname{Men}(n, \%)$ & $189(58)$ \\
\hline \multirow[t]{2}{*}{ Age, years } & Mean (SD) & $69( \pm 12)$ \\
\hline & Age $\geq 80(n, \%)$ & $80(24.7)$ \\
\hline \multirow{6}{*}{ Primary cancer $(n, \%)$} & Lung & $71(21.9)$ \\
\hline & Colon & $38(11.7)$ \\
\hline & Pancreas & $28(8.6)$ \\
\hline & Breast & $22(6.8)$ \\
\hline & Prostate & $18(5,6)$ \\
\hline & Other origins & $146(45.1)$ \\
\hline \multirow[t]{2}{*}{ Extension of cancer $(n, \%)$} & Local and regional & $47(14.6)$ \\
\hline & Metastasis & $276(85.4)$ \\
\hline \multirow{3}{*}{$\begin{array}{l}\text { Cancer treatment in } 4 \text { weeks before inclusion } \\
(n, \%)\end{array}$} & Chemotherapy & $169(52.3)$ \\
\hline & Radiotherapy & $87(26.9)$ \\
\hline & Hormone therapy & $16(4.9)$ \\
\hline Barthel index & Mean (SD) & $60.5( \pm 26)$ \\
\hline \multicolumn{2}{|l|}{ Karnofsky scale $\leq 50 \%(n, \%)$} & $129(39.8)$ \\
\hline Symptom burden & $(n, \%)$ & Mean (SD) NRS \\
\hline Asthenia & $299(92.3)$ & $5.7(2.1)$ \\
\hline Anorexia & $253(78.1)$ & $5.4(2.1)$ \\
\hline Pain & $245(75.6)$ & $3.7(2.6)$ \\
\hline Nausea/vomiting & $110(34.0)$ & $4.1(1.9)$ \\
\hline Constipation & $202(62.3)$ & $4.5(1.9)$ \\
\hline Breathlessness & $149(45.9)$ & $4.5(1.9)$ \\
\hline Insomnia & $191(58.9)$ & $2.7(2.1)$ \\
\hline Anxiety & $238(73.4)$ & $4.6(2.1)$ \\
\hline Depression & $225(69.4)$ & $4.7(1.9)$ \\
\hline Number of symptoms/patient & Mean (SD) & $5.9( \pm 1.2)$ \\
\hline \multirow{2}{*}{$\begin{array}{l}\text { Number of symptoms with NRS } \geq 4 \text { /patient } \\
\text { Patients with } \geq 5 \text { symptoms with NRS } \geq 4\end{array}$} & Mean (SD) & $3.1( \pm 1)$ \\
\hline & $(n, \%)$ & $87(26.8)$ \\
\hline \multicolumn{3}{|l|}{ Type of pain } \\
\hline Nociceptive somatic & $(n, \%)$ & $170(52.8)$ \\
\hline Nociceptive visceral & $(n, \%)$ & $94(29.2)$ \\
\hline Neuropathic & $(n, \%)$ & $60(18.6)$ \\
\hline Breakthrough pain & $(n, \%)$ & $153(47.5)$ \\
\hline Pain in addictive behavior & $(n, \%)$ & $11(3.4)$ \\
\hline Pain stage II of ECS-CP & $(n, \%)$ & $175(54.0)$ \\
\hline Cognitive impairment, (CAM: positive) & $(n, \%)$ & $25(7.7)$ \\
\hline Sociofamilial risk factors & $(n, \%)$ & $222(68.5)$ \\
\hline Support limited by advanced caregiver age & $(n, \%)$ & $84(25.9)$ \\
\hline Support limited by high workloads or family & $(n, \%)$ & $69(21.3)$ \\
\hline Absence of family or identified caregiver & $(n, \%)$ & $34(10.5)$ \\
\hline Other social risk factors & $(n, \%)$ & $34(10.8)$ \\
\hline
\end{tabular}

NRS, Numerical Rating Scale; ESS-CP, Edmonton Classification Scale for Cancer Pain; CAM; Confusion Assessment Method. 
Table 2: Prevalence of ethical dilemmas overall, by category, and by patient characteristics.

\begin{tabular}{|c|c|c|c|c|c|c|c|c|c|c|c|}
\hline & & $n$ & $\%$ & $\begin{array}{l}\text { Male sex } \\
\text { N (\%) }\end{array}$ & $p$ & $\begin{array}{c}\text { Chemotherapy } \\
(n, \%)\end{array}$ & $p$ & $\begin{array}{l}\text { Functional } \\
\text { impairment: } \\
(n, \%)\end{array}$ & $p$ & $\begin{array}{l}\text { Emotional } \\
\text { distress: } \\
(n, \%) \\
\end{array}$ & $p$ \\
\hline \multicolumn{2}{|l|}{ Total $n$} & 324 & & & & & & & & & \\
\hline \multicolumn{2}{|c|}{$n$ with $\geq 1$ ethical dilemma } & 90 & 27.8 & $60(66.7)$ & $\begin{array}{c}<0.00 \\
2\end{array}$ & $55(61.1)$ & $\begin{array}{c}<0.03 \\
5\end{array}$ & $53(58.9)$ & 0.091 & $66(73.3)$ & $\begin{array}{c}<0.00 \\
1\end{array}$ \\
\hline \multicolumn{2}{|c|}{ Total $n$ of dilemmas } & 117 & & & & & & & & & \\
\hline \multicolumn{2}{|c|}{ Conflicts associated with information } & 51 & 15.7 & $28(54.9)$ & 0.438 & $26(50.9)$ & 0.886 & $26(50.9)$ & 0.886 & $29(56.9)$ & 0.327 \\
\hline \multirow[t]{4}{*}{$\begin{array}{l}\text { Therapeutic } \\
\text { proportionality }\end{array}$} & $\begin{array}{l}\text { Discrepancies: } \\
\text { Within the } \\
\text { family }\end{array}$ & $\begin{array}{l}54 \\
26\end{array}$ & $\begin{array}{c}16.7 \\
8.0\end{array}$ & $11(42.3)$ & 0.433 & $15(57.7)$ & 0.432 & $0(0)$ & - & $15(57.7)$ & 0.432 \\
\hline & $\begin{array}{l}\text { Between the } \\
\text { care team and } \\
\text { the } \\
\text { patient/family }\end{array}$ & 14 & 4.3 & $8(57.1)$ & 0.590 & $8(57.1)$ & 0.590 & $12(85.7)$ & 0.075 & $12(85.7)$ & 0.075 \\
\hline & $\begin{array}{l}\text { Within the care } \\
\text { team }\end{array}$ & 12 & 3.7 & $5(41.7)$ & 0.567 & $3(25.0)$ & 0.083 & $7(58.3)$ & 0.563 & $3(25.0)$ & 0.083 \\
\hline & $\begin{array}{l}\text { With respect to } \\
\text { withdrawal of } \\
\text { life support }\end{array}$ & 2 & 0.6 & $1(50.0)$ & - & $0(0)$ & - & $2(50.0)$ & - & $0(0)$ & - \\
\hline \multirow{2}{*}{\multicolumn{2}{|c|}{$\begin{array}{l}\text { Conflicts associated with the expected } \\
\text { outcomes of a clinical trial. } \\
\text { Request for euthanasia/assisted suicide }\end{array}$}} & 8 & 2.5 & $5(62.5)$ & 0.475 & $2(25.0)$ & 0.157 & $4(50.0)$ & - & $6(75.0)$ & 0.157 \\
\hline & & 4 & 1.2 & $2(50.0)$ & - & $1(25.0)$ & 0.317 & $2(50.0)$ & - & $1(25.0)$ & 0.317 \\
\hline
\end{tabular}

